Même les pays du Marché Commun ne peuvent atteindre pour l'instant le niveau de consommation des États-Unis : la consommation européenne de viande bovine ne progresse plus guère. Le Marché Commun qui importe de la viande bovine et qui exporte des produits laitiers éprouve des difficultés à assurer ses approvisionnements en viande sans augmenter inconsidérément ses excédents laitiers : les rendements en lait augmentent plus vite que les rendements en viande.

La production de viandes de volailles et de porcs, les races bovines à viande, les croisements industriels et la finition accélérée des jeunes bovins devront donc aussi contribuer à combler le déficit. Mais il faudra aussi compter de plus en plus sur la mobilisation des ressources fourragères des régions de montagne et sur celle des sous-produits agricoles des régions de grande culture et de l'industrie. Ce déséquilibre croissant peut aussi être comblé par le développement de la consommation directe de protéines végétales soumises à des traitements industriels.

Si ces perspectives apparaissent de plus en plus clairement, il n'en reste pas moins que les prévisions à long terme et détaillées qui pourraient permettre d'orienter les travaux de recherche et d'amélioration génétique appliqués aux animaux domestiques, sont pratiquement impossibles dans la situation de grande instabilité de l'économie mondiale. Quant aux résultats des études coât-bénéfice, ils sont aussi incertains que les hypothèses de prix et de taux d'actualisation qui les sous-tendent.

D'une manière générale, la recherche doit fournir une gamme de techniques et une gamme de résultats assez étendue pour répondre aux besoins économiquement et socialement très diversifiés des différentes catégories de producteurs et pour répondre à des situations économiques à venir en grande partie imprévisibles. La recherche et la mise au point des techniques dans une société doivent pouvoir jouer un rôle contre-aléatoire important, pour donner à l'appareil de production toute la souplesse de fonctionnement et toute l'adaptabilité qui lui sont nécessaires. Elles doivent aussi donner à toutes les catégories de producteurs en place les moyens de tirer le meilleur parti des ressources qu'ils contrôlent socialement et qu'ils exploitent.

Dans ces conditions, la meilleure voie de collaboration qui puisse exister entre économistes et spécialistes de la production animale, consisterait à créer pour chaque région d'élevage, des équipes mixtes capables d'étudier la situation, l'avenir, les conditions, les problèmes de développement et les besoins de chaque grand groupe de producteurs existant aujourd'hui.

Ainsi, pourrait-on espérer tirer le meilleur parti des ressources naturelles et du très riche héritage de l'élevage européen.

Pour procéder de la sorte, il faudra sans doute, dans la plupart des pays, augmenter considérablement les moyens de la recherche au niveau régional.

\title{
ZUCHTZIELSETZUNG FÜR ZWEINUTZUNGSRINDER
}

\section{Fewson. - Abteilung für Tierzüchtung Universität Hohenheim Landwvirtschaftliche Hochschule 7 Stuttgart 70, Postfach 106 (BRD).}

I. Sofern die relative wirtschaftliche Bedeutung der Eiweissmenge im Vergleich zur Fettmenge weiter ansteigt, sollte die Eiweissmenge zusätzlich in den Gesamtzuchtwert aufgenommen werden.

2. Die Variation des Anteiles der wertvollen Teilstücke - Keule, Roastbeef mit Filet und Hochrippe - ist so gering, dass mit diesem Merkmal die Schlachtkörperqualität von Mastrindern nicht hinreichend erfasst werden kann. Für diesen Zweck wird vorgeschlagen, den Fleischanteil des Mastrindes als Leistungsmerkmal zu verwenden. Der Fleischanteil des Mastrindes entspricht dabei der Fleischmenge des Schlachtkörpers. Bei einer solchen Betrachtungsweise sind dann die Schlachtausbeute, der Fettanteil, der Knochenanteil und eine subjektive Bewertung als Hilfsmerkmale für den Fleischanteil zu betrachten.

3. In weiteren Untersuchungen ist zu prüfen, ob durch Einbeziehung der Leichtkalbigkeit und der Melkbarkeit in den Gesamtzuchtversuch die monetäre, genetisch nutzbare Variation nachhaltig gesteigert werden kann.

4. Nach dem heutigen Wissensstand erscheint es nicht möglich, alle Merkmale, die sich auf die Rentabilität der Nutztierproduktion direkt oder indirekt auswirken, in einen Gesamtzuchtwert einzubeziehen. Für eine Reihe von Merkmalen - wie z. B. Klauenmängel, Euter- und Zitzenform usw. - fehlen die erforderlichen ökonomischen und genetischen Parameter. Neben der Selektion nach einem Gesamtzuchtwert wird deshalb in der Zuchtpraxis eine Zuchtwahl nach diesen noch nicht quantifizierbaren Merkmalen ablaugen. Dies ist bei Zuchtplanungen durch angemessene Selektionsraten zu berücksichtigen.

5. Mit diesem Referat kann lediglich ein Beitrag zur Methodik der Aufstellung von Gesamtzuchtwerten geliefert werden. Die Zuchtzielsetzung durch Quantifizierung des Gesamtzuchtwertes 
ist eine Aufgabe der Zuchtleitung jeder einzelnen Population. Dabei müssen die vorherrschenden Produktionsbedingungen durch entsprechende Wirtschaftlichkeitskoeffizienten und zusätzlich die populationsspezifischen genetischen Parameter der Leistungsmerkmale berücksichtigt werden. Von der Wissenschaft können hierfür lediglich Berechnungsansätze bereitgestellt werden.

\title{
L'AMÉLIORATION GÉNÉTIQUE DE LA PRODUCTION DE VIANDE, DANS LES TROUPEAUX SPÉCIALISÉS ET PAR CROISEMENTS DANS LES TROUPEAUX LAITIERS
}

\author{
F. Menissier, B. Vissac, J. Frebling. - Département de Génétique animale, C. N.R.Z., \\ 78350 Jouy en Josas (France) (1).
}

Dans les prochaines années, compte tenu de la réduction du nombre de vaches laitières en Europe, l'augmentation de la production de viande se fera par un accroissement du croisement avec des taureaux de races à viande dans les élevages laitiers et par un développement des troupeaux de vaches allaitantes à partir des femelles éliminées de la traite. Dans ce processus général d'évolution dans l'utilisation du matériel génétique où la production de viande apparaît comme un sous-produit de la sélection laitière, la situation de chaque pays varie selon ses caractéristiques agronomiques, climatiques et structurelles, les besoins de son marché et les potentialités de son matériel génétique. Les situations de production de viande seront donc nombreuses pour l'Europe.

Le développement de stratégies de croisement à partir des cheptels de femelles existants et de races paternelles ayant des aptitudes complémentaires, se justifie et s'explique :

- par la spécificité des fonctions exploitées d'une part chez la femelle laitière ou allaitante, d'autre part chez les produits destinés à la boucherie ;

- par les oppositions fréquentes entre la contribution des gènes de la mère et celle des gènes du produit pour un caractère donne ;

- et par l'importance des effets d'hétérosis.

Les stratégies de croisement envisageables se déterminent en fonction du type d'exploitation des mères et du matériel génétique disponible. Les objectifs de choix des races paternelles (ou types génétiques ou souches) vont alors dépendre des stratégies retenues ainsi que de l'utilisation des produits femelles issus de ces races paternelles (abattage comparable à celui des mâles de boucherie, abattage après un premier vêlage précoce, exploitation comme vaches allaitantes assurant ou non leur renouvellement). Dans un contexte donné, les solutions optimum à promouvoir doivent découler d'études de synthèse prenant en compte notamment les restrictions et équilibres indispensables entre les composantes maternelles et paternelles de certains caractères (aptitude au vêlage et croissance avant sevrage).

Pour réaliser de telles études de synthèse, notre connaissance du matériel génétique existant est très insuffisante en ce qui concerne les contributions additives et non additives des gènes de la mère et du produit tant sur les caractères de productivité numérique que sur ceux de productivité pondérale. Les expériences réalisées en Europe sont trop limitées dans leur durée, dans la diversité du matériel génétique employé et dans le niveau d'analyse génétique, pour répondre en temps opportun et de façon efficace aux choix qui se posent dans la plupart des pays. Ainsi, les modèles de synthèse actuellement employés ignorent souvent les différences de productivité numérique entre races ainsi que l'incidence de la musculature sur la définition des âges optimum d'abattage et sur la valeur bouchère des vaches de réforme. Incontestablement, la notion de race devrait être dépassée dans de telles expériences et analyses, compte tenu de la diversité des souches existantes dans la plupart de nos races (Charolaise, Pie-Noire, Simmental, Brune des Alpes). Une action commune (ou tout au moins concertée) à partir des populations de femelles laitières et sur la base des schémas expérimentaux proposés par Dickerson (I969) serait du plus haut intérêt en Europe.

Par ailleurs, dans la mesure où l'insémination artificielle est très développée en Europe, les schémas intégrés de sélection des mâles (noyau de femelles, contrôle individuel puis contrôle de descendance) sont très efficaces et fournissent l'information nécessaire à l'amélioration de la productivité. Ces schémas de sélection constituent des situations dans lesquelles les nouvelles techniques de reproduction (transplantation d'œufs en particulier) vont être efficacement rentabilisées même en l'état actuel de ces techniques ; un accroissement notable du progrès génétique

() Ce texte résume la plaquette en anglais des mêmes auteurs : Optimun breeding plans for beef cattle. Bull. tech. Dép. Genét. anim. (I. N. R. A., Fr.) no 21,102 p. 